\title{
A study of stress levels amongst patients undergoing third molar extraction by local and general anaesthesia
}

\author{
Salivary cortisol determinations and self-rating scales in the assessment of stress in patients undergoing the extraction of \\ wisdom teeth by C. M. Hill and R. V. Walker Br Dent J 2001; 191:513-515
}

\section{Objective}

To investigate salivary cortisol levels in patients undergoing third molar removal under local and general anaesthesia.

\section{Design}

Random sampling of two independent groups.

\section{Methods}

Samples of saliva were collected from patients prior to and after surgery for the removal of impacted wisdom teeth. The samples were analysed to assess cortisol levels. Patients were also asked to complete a Hospital Anxiety and Depression questionnaire on the day of surgery.

\section{Results}

Patients receiving treatment under local anaesthesia showed lower levels of stress response than those having treatment under general anaesthesia on the actual day of surgery did (Fig. 1). No other significant differences were noted.

\section{Conclusion}

Local anaesthesia is the preferred mode of pain control for patients who express no strong preference for type of anaesthetic.
In Brief
- Salivary cortisol assays are useful in assessing the stress response within a population of surgical patients.
- There is no evidence that having wisdom teeth removed under general anaesthesia is less stressful than under local anaesthesia.

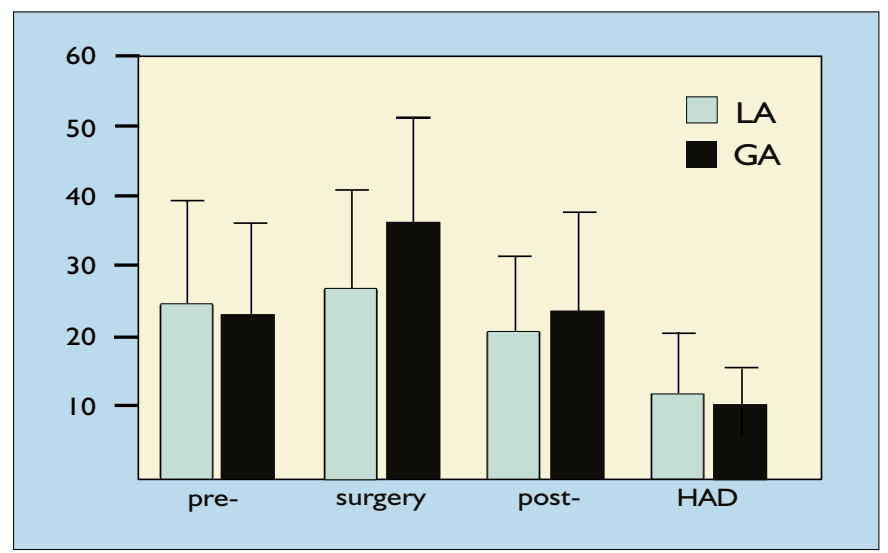

Fig. I Mean + I S.D. salivary cortisol concentrations (nmol/L) pre-, post- and on the day of surgery in subjects undergoing extraction of wisdom teeth under either general anaesthetic (GA; $n=27)$ or local anaesthetic (LA; $n=27)$.

\begin{abstract}
Comment

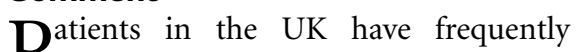
demanded general anaesthesia for dental treatment but stringent regulation imposed by the General Dental Council in 1998 has accelerated the reduction in the number of dental general anaesthetics administered. General anaesthesia is associated with significant morbidity and is never without risk. A reduction in its use for dental treatment is recognised as a positive advantage in the provision of quality healthcare. However, there will always be a proportion of the population for whom general anaesthesia is appropriate and whilst the use of general anaesthesia has been excessive in the UK, some other countries have inadequate access.

The choice of anaesthesia is based on assessment of the anticipated difficulty of the procedure, the medical and social history, and the psychological needs of the individual patient. This paper looks at a method for measuring stress associated with the local or general anaesthesia provided for third molar surgery as a basis for
\end{abstract}

comment about the usefulness of the technique and also the choice of anaesthesia.

Salivary cortisol levels are know to correlate with psychological stress and have been used to assess stress in a variety of individuals, such as examination students and air traffic controllers. The authors used this method to measure cortisol levels on 3 days before surgery, the morning of surgery and the following 3 days. The technique was found to be useful and particularly valuable because of its non-invasive nature.

Patients in the general anaesthesia group showed a significant increase in salivary cortisol on the morning of surgery compared with their pre-operative and postoperative measures. Patients in the local anaesthetic group showed a slight increase in mean salivary cortisol but this was not statistically significant. The authors suggest that the anticipation of general anaesthesia produces more stress than the anticipation of local anaesthesia and conclude that avoidance of this stress is a reason to prefer local anaesthesia. However, the patients in this study were not allocated randomly to groups but rather according to their own choice. It is possible that the more anxious patients opted for general anaesthesia and this is why this group showed more stress preoperatively compared with the less anxious patients who opted for local anaesthesia. Perhaps the more anxious patients would have shown even more stress if they were anticipating surgery under local anaesthesia. By allocating the participants randomly, the characteristics of the patients would have been similar across groups at the start of the comparison, and the investigators would have been more able to quantify the impact of the anaesthetic choice, with minimal effects from other factors. It would have been interesting to assess patient satisfaction with the choice of anaesthesia.

\section{Paul Coulthard}

Senior Lecturer and Honorary Consultant, Head of Oral and Maxillofacial Surgery, University Dental Hospital of Manchester 